\title{
Coherence properties and diagnostics of betatron radiation emitted by an externally-injected electron beam propagating in a plasma channel
}

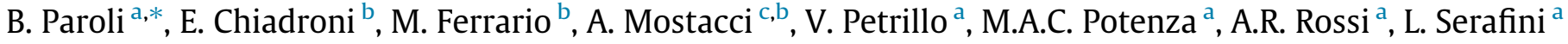 \\ a Dipartimento di Fisica, Universitá degli Studi di Milano and INFN Sezione di Milano, via G. Celoria, 16, 20133 Milano, Italy \\ ${ }^{\mathrm{b}}$ INFN-LNF, via E. Fermi, 00044 Frascati, Italy \\ c “La Sapienza” University, SBAI Department, via A. Scarpa 14, 00161 Rome, Italy
}

\section{A R T I C L E I N F O}

\section{Article history:}

Received 27 November 2014

Received in revised form 20 March 2015

Accepted 24 March 2015

Available online 3 April 2015

\section{Keywords:}

Plasma acceleration

X-ray diagnostics

Betatron radiation

\begin{abstract}
A B S T R A C T
A 3-dimensional time-domain simulation of X-ray produced by a laser wakefield accelerated electron beam was performed in order to know its properties like intensity, spectrum, divergence and coherence. Particular attention was paid to the coherence around the acceleration axis. The broad spectrum of betatron radiation (1-10 keV) leads to a short coherence length. Nevertheless we observe that under particular detection condition the spatial coherence has a characteristic enlargement. We give a simplified interpretation of this effect in terms of phase shift of the electric field on a virtual detector. Moreover we describe a near field scattering technique to characterize the betatron radiation. This diagnostics will be used to map the transverse spatio-temporal coherence of X-ray radiation in the laser wakefield accelerator under development at Frascati National Laboratories (LNF).
\end{abstract}

(c) 2015 Elsevier B.V. All rights reserved.

\section{Introduction}

Laser wakefield acceleration has recently been exploited also to produce femtosecond X-ray bursts with low divergence and high brightness [1,2]. Simulation and detection of such radiation are of relevant interest because they can give theoretical and practical information about the beam properties in beam-plasma interaction $[3,4]$. In this work we present recent results about a 3-dimensional time-domain simulation of the radiation emitted by a low emittance $(2.7 \mathrm{~mm}$ mrad) $14 \mathrm{pC}$ electron beam with $3.5 \mu \mathrm{m}$ size and $78 \mathrm{MeV}$ initial energy (these parameters match the SPARC electron beam parameters at LNF). The code computes the Lienard-Wiechert potentials in a space-time matrix of 268 Mpixel, starting from the electron trajectories produced by the Q-fluid code [5]. Plasma wave is generated by means of a 100 TW, 35 fs laser pulse compatible with the laser FLAME [6] and the plasma evolution is computed using the fluid equations in cylindrical symmetry. Electron trajectories of an externally injected bunch are solved integrating the equations of motion in the electromagnetic field produced by both laser and plasma. Particular attention was paid to the coherence properties around the acceleration axis. In fact the broad spectrum of betatron radiation limits the coherence length. Thus the transverse size of the

\footnotetext{
* Corresponding author.

E-mail address: bruno.paroli@unimi.it (B. Paroli).
}

coherence area is also limited. In principle, to circumvent such limitation a proper curved detector is required rather than a planar detector so that the extra-path $\delta_{L}=L^{\prime}-L$ is at least reduced for a localized source. Here $L^{\prime}$ is the distance between the bunch and an arbitrary off-axis point of the detector, while $L$ is the bunch-detector distance along the acceleration axis. In such condition an enlargement of the coherence area is significantly observed. This phenomenon can be interpreted studying the phase shift of the simulated electric field on the curved detector as a function of the observation angle. Moreover we discuss about the optical diagnostics under development at the University of Milan in collaboration with Frascati National Laboratories to detect the X-ray radiation emitted by electron during acceleration. The diagnostics uses a near field scattering (NFS) of the incident radiation from micro particles in heterodyne condition to map the spatio-temporal coherence of betatron radiation. The paper is organized as follows. In Section 2 we show the simulation results and discuss the observed coherence enlargement. Section 3 is devoted to the NFS diagnostics. Finally in Section 4 we draw our conclusions.

\section{Simulation method and results}

A simulation of the X-ray radiation emitted by the externally injected electron bunch accelerated in a $10^{17} \mathrm{~cm}^{-3}$ plasma channel was performed in time domain. A slice of $1 / 20$ of the bunch length was selected from the $2 \cdot 10^{4}$ electron trajectories. The computing 
time was about 3 days to solve a matrix of $64 \times 64$ elements in space and $2^{16}$ elements in time. In such conditions we reach a time step of $\Delta t=10^{-4}$ fs over a maximum time evolution of $6.5 \mathrm{fs}$. The points trajectory of each particle computed by Q-fluid were increased of a factor 100 by means of a spline interpolation. This step is needed to obtain smoother trajectories and to reduce the error of the first derivative of the electron momentum in calculation.

Electric $\vec{E}(i, j, t)$ and magnetic $\vec{B}(i, j, t)$ fields are solved at the retarded time $t^{\prime}$ by means of the Lienard-Wiechert formulas at each pixel $(i, j)$ of the virtual detector and for each particle. The fields are then converted in the lab frame time $f\left(t^{\prime}\right)=t$ using the derivative $d f\left(t^{\prime}\right) / d t=1-\vec{n} \cdot \vec{\beta}$, where $\vec{n}$ is the unit vector directed from the particle to the observer and $\vec{\beta}=\vec{v} / c(\vec{v}$ is the particle velocity and $c$ is the speed of light). The conversion from the retarded time to the lab frame time $t=\int f\left(t^{\prime}\right) d t$ introduces a non-uniform time step. The uniformity is than restored with a linear interpolation using an adaptive scheme: the time step of the uniform grid is changed as a function of the angle of view of the emitted radiation. The advantage of this method is that we can properly match the time resolution with the characteristic times of variation $\Delta t=2 \pi / \omega_{c}$ of the emitted electromagnetic field that change appreciably moving from the acceleration axis as $\Delta t \approx 4 \pi \theta_{e}^{2} c / 3 a_{\beta}^{2} \omega_{p}^{2} r_{\beta}$, where $\omega_{p} / 2 \pi$ is the plasma frequency, $r_{\beta}$ is the betatron oscillation amplitude, $a_{\beta}$ and $\theta_{e}$ are the wiggler parameter and the radiation emission angle, respectively. We show in Figs. 1-3 the simulation results relative to intensity, divergence and spectrum calculated at a distance of $1 \mathrm{~m}$ from the beam initial position. The total energy is approximatively $3 \cdot 10^{-2} \mathrm{fJ} /$ electron with a spectrum in the soft-X-ray range (1-10 keV) and with divergence of about $10 \mathrm{mrad}$. Simulation confirms that X-ray produced by externally injected bunch have a similar behavior of those generated in a self-injection scheme [7].

The advantage to compute $\vec{E}$ and $\vec{B}$ in time domain is that we draw the whole information about radiation, including the wavefront phase of the electromagnetic field on the virtual detector. The coherence length $L_{c}$ of radiation close to the critical frequency $\omega_{c} \hbar \approx 1 \mathrm{keV}$ is about $1.2 \mathrm{~nm}$. Even though the coherence length is so short its effect is important in determining the resulting size of the coherence area. We expect that the transverse dimension $S_{c}$ of the coherence area, for a radiation with limited coherence length $\left(\lambda^{2} / 2 \Delta \lambda \approx \lambda\right)$ is of the order of

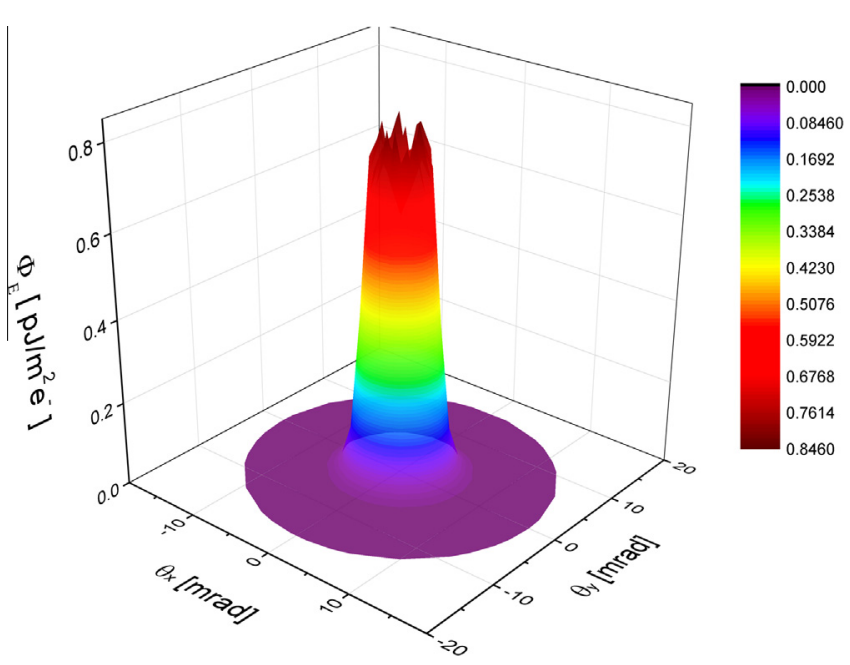

Fig. 1. Irradiance of the betatron radiation measure on-axis at a distance of $1 \mathrm{~m}$ from the beam initial position.

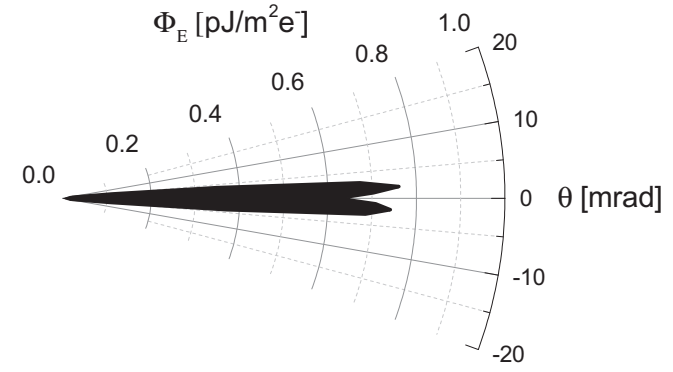

Fig. 2. Polar diagram of the irradiance (see Fig. 1).

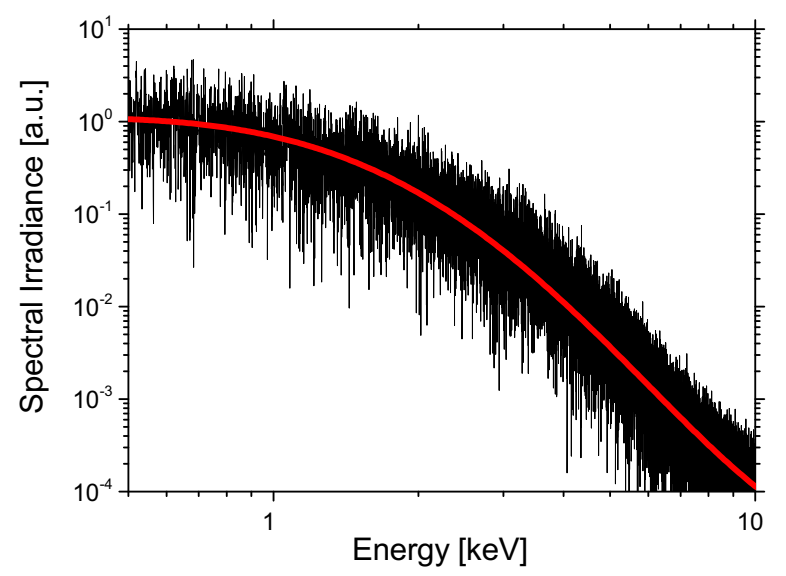

Fig. 3. Spectral irradiance measured on-axis. The red line is a polynomial fit of the simulated spectral irradiance (black). (For interpretation of the references to color in this figure caption, the reader is referred to the web version of this article.)

$S_{c}=L \cdot \arccos \frac{L / L_{c}}{1+L / L_{c}}$

where $L$ is the beam-detector distance. The transverse size of the coherence area is defined here as the Full Width Half Maximum (FWHM) of the coherent factor

$\gamma_{c}=\frac{\Gamma\left(\overrightarrow{X_{0}}, \vec{X}\right)}{\left[\Gamma\left(\overrightarrow{X_{0}}, \overrightarrow{X_{0}}\right) \Gamma(\vec{X}, \vec{X})\right]^{1 / 2}}$

where $\Gamma\left(\overrightarrow{X_{0}}, \vec{X}\right)=\left\langle E\left(X_{0}\right) E^{*}(X)\right\rangle, E$ is the complex representation of the electric field. The FWHM of the simulated coherence area is $44 \mu \mathrm{m}$ in good agreement with the calculated value $S_{c}=50 \mu \mathrm{m}$ (see Eq. (1)). A curved detector with radius $L$ would compensate the extra-path $\left(\delta_{L}=L^{\prime}-L\right)$ and the size of the coherence area becomes $200 \mu \mathrm{m}$ : four times than that measured with the planar detector. This enlargement of the coherence area was investigated studying the phase shift of the electric field on the virtual detector surface. We note that the $x$ component of the $\vec{E}$ field, observed at $\theta=0$, where $\theta$ is the colatitude angle of observation, is delayed with respect to the fields measured at lower angles $\theta<0.1 \mathrm{mrad}$, while it appears earlier than the fields measured at higher angles, i.e. $\theta>0.14$ (see Fig. 4). The field at $\theta=0$ must therefore overlaps in time with the field observed at an angle of the order of $0.1 \mathrm{mrad}$. This particular angle is in very good agreement with the dimensions of the coherence area size, $2 L \tan \theta$. Our preliminary interpretation is that for the radiation emitted by each particle in a narrow cone along $\vec{\beta}$, the curved trajectory determines the phase shift of the wavefront across the virtual detector. Works are in progress to enforce this interpretation. 


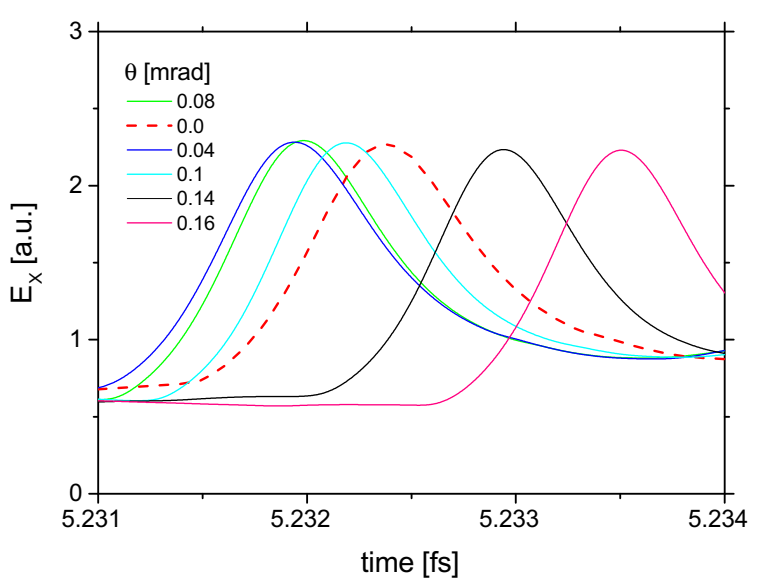

Fig. 4. Phase shift of the electric field ( $x$ component) measured at different angles of observation $\theta$. The field is calculated on a curved detector at a distance of $1 \mathrm{~m}$ from the beam initial position. The red dashed line is the reference field measured along the acceleration axis. (For interpretation of the references to color in this figure legend, the reader is referred to the web version of this article.)

\section{Near field scattering diagnostics}

The near field scattering (NFS) technique was used successfully to map the spatial coherence in synchrotron [8] and in free electron laser radiations [9]. We describe in this section the diagnostics under development at the University of Milan to measure the coherence of radiation emitted by laser wakefield acceleration. A sketch of the diagnostic is shown in Fig. 5. Betatron radiation illuminates a scattering cell of randomly distributed micro-particles (with an average radius in the range $0.2-1 \mu \mathrm{m}$ ) suspended in liquid (generally water). The fields diffracted from each particle overlap in space producing a very complex speckle field. The intensity of the field is not deterministic because both the random distribution and the brownian motion of particles. The low concentration of solution generally between $10^{-4}$ and $10^{-5} \mathrm{vol}$. together with a relatively thin cell $(1-2 \mathrm{~mm})$ determines a faint scattered field and an intense transmitted radiation $(\approx 95 \%)$. This is essential to reach the heterodyne condition in which the interference between the transmitted and the scattered fields dominates on the cross interference. Under such condition, the system behaves like a self-referenced interferometer, where the reference is represented here by each scatter wave, which determines a system of fringes that inherits the properties of the illuminating beam. Even though the complexity of the resulting intensity distribution in space, the analysis of the speckle field in the spatial frequencies domain has a direct interpretation. The power spectrum $S(\vec{q}, z, t)$ can be factorized as:

$S(\vec{q}, z, t)=\Phi(\vec{q}) C(\vec{q}, z) T(\vec{q}, z)$

where $\Phi(\vec{q}, t)$ is the form factor of the ensemble of scatters including scaling constants, $T(q)=\sin ^{2}\left(z q^{2} / 2 k\right)$ is the Talbot transfer function, and $C(q)$ is the squared modulus of the coherence factor $\gamma_{c}$. The coherence mapping by means of the variable $\vec{q}=k \vec{\Delta} r / z$ occurs because are experimentally known the functions $\Phi(\vec{q})$ and $T(\vec{q}, z)$. Here $\vec{\Delta} r$ is the position vector of the detector point, $z$ is the cell-detector distance and $k=2 \pi / \lambda$.

As discussed in Section 2, the temporal coherence of betatron radiation dominates when the coherence area is characterized on a planar detector. The expected coherence area has a dimension of about $50 \mu \mathrm{m}$ at a distance of $1 \mathrm{~m}$ along the acceleration axis. Thanks to the angular dependence of the Lorentz factor $\gamma$ and the wiggler parameter $a_{\beta}$ on the emission angle $\theta_{e}=a_{\beta} / \gamma$, a measurement of the coherence provides the characterization of the critical frequency $\omega_{c} \approx 3 a_{\beta}^{3} \gamma^{2} \omega_{\beta} / 4$ as a function of $\theta_{e}$, where $\omega_{\beta} / 2 \pi$ is the betatron frequency. This is also a characterization of the acceleration process through the parameter $\omega_{c} \approx 3 \omega_{p}^{2} r_{\beta} \gamma^{2} / 2 c$, with a resolution limited principally by the $1 / \gamma$ angle. Another application of such diagnostics is the systematic investigation in two-dimensions of the spatial coherence in a limited spectrum around at a given central frequency $\omega_{0}$. The narrowing of the band can be obtained by using a monochromator. As established in Refs. [8,9] the diagnostics is able to operate from visible light to X-ray radiations with a possible extension to the infrared region. The diagnostics hardware is relatively simple and free of any X-ray optics. It includes: a 2-dimension positioner that moves the scattering cell transversally to the beam propagation axis; a 3 dimensional positioner that moves a CCD camera equipped with a beryllium high pass filter and a P45 scintillator; a timing system to synchronize the image acquisition with the laser shot. The drivers of the

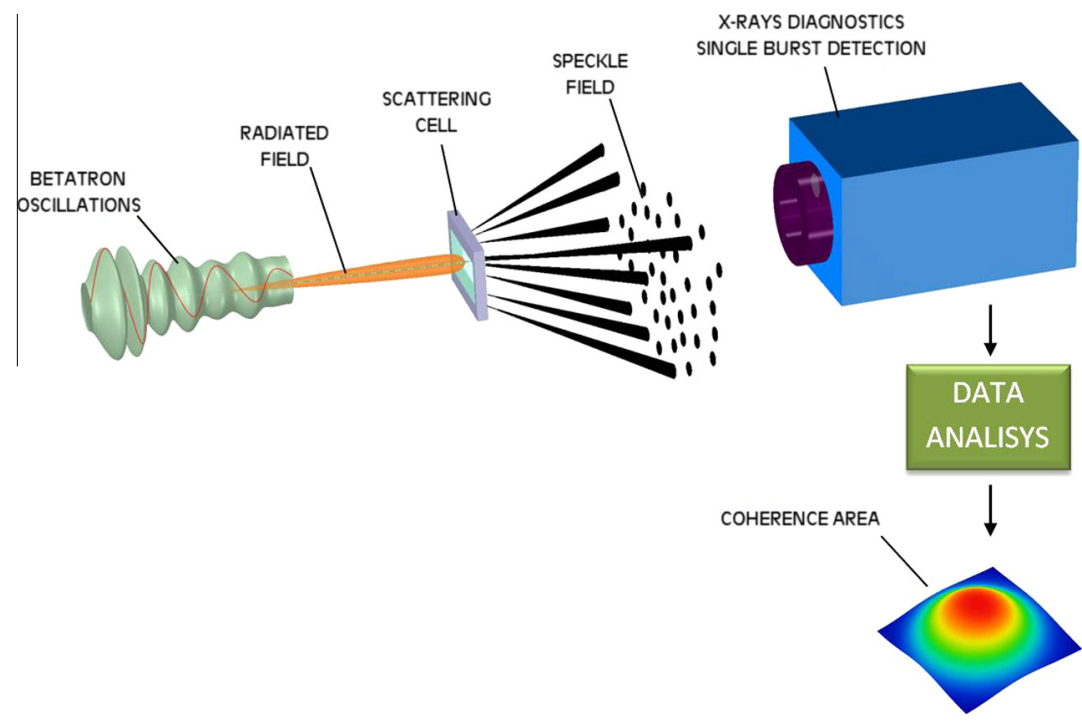

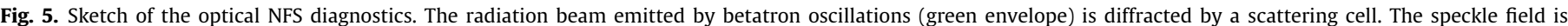

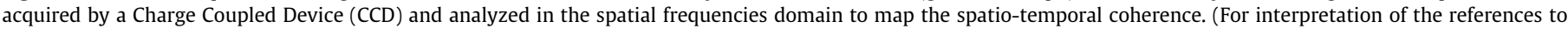
color in this figure legend, the reader is referred to the web version of this article.) 
acquisition system and positioners will be controlled remotely by a server unit.

\section{Conclusions}

We have shown in this paper the results of a simulation based on the Lienard-Wiechert formulas for an externally injected electron bunch accelerated by a plasma wakefield. The intensity and divergence of the X-ray radiation are roughly comparable with that of the self-injected scheme. A particular study was dedicated to coherence properties which are (on a planar virtual detector) compatible with a temporal coherence dominated radiation. Compensating the coherence length by mean of a curved detector we would observe that the $50 \mu \mathrm{m}$ coherence area size increases to $200 \mu \mathrm{m}$. Such enlargement is preliminarily described as an effect of the electric field phase shift depending on the angle of observation. It can be experimentally measured using a double pinhole interferometer so that a pinhole can be moved along a curved path corresponding with the points of the curved virtual detector of radius $L$. A main limitation of such approach is given by the high variability of the betatron radiation pulses. In fact each point of the coherence map requires at least one shot. Finally we have discussed the use of the heterodyne near field scattering diagnostics as a method to map the spatio-temporal coherence of betatron radiation in broad or narrow spectrum conditions (from X-ray to infrared). In the broad spectrum case the coherence area is a measurement of the coherence length (see Eq. (1)), i.e. a measurement of the critical frequency $\omega_{c} \sim 2 \pi c / L_{c}$ of the radiation.

\section{Acknowledgments}

This work was supported by the Italian Ministry for University and Research (MIUR) "FIRB 2012" funds (Grant No. RBFR12NK5K).

\section{References}

[1] S. Corde et al., Rev. Mod. Phys. 85 (2013).

[2] S. Kneip et al., Nat. Phys. 6 (2010) 980-983.

[3] K. Phuoc et al., Phys. Rev. Lett. 97 (2006) 225002.

[4] M. Schnell et al., Phys. Rev. Lett. 108 (2012).

[5] A. Rossi et al., Nucl. Instr. Meth. Phys. Res. A 740 (2014) 60-66.

[6] T. Levato et al., Nucl. Instr. Meth. Phys. Res. A 720 (2013) 95-99.

[7] A. Rousse et al., Phys. Rev. Lett. 93 (2004).

[8] M. Alaimo et al., Phys. Rev. Lett. 103 (2009).

[9] M. Alaimo et al., Opt. Express 22 (2014). 